\title{
Die Schweiz, Plattform der Zigarettenhersteller
}

\section{Rainer M. Kaelin}

Dr. med., Facharzt Innere Medizin und Pneumologie, Mitglied FMH

\section{Einleitung}

Das Bundesparlament wird mit dem Tabakproduktegesetz (TabPG) die Regeln um ein toxisches Konsumgut neu festlegen. Weder die Verfechter der freien Marktwirtschaft noch die der Prävention sind mit dem Entwurf zum TabPG glücklich: Für die einen gehen die Eingriffe für ein legal verkäufliches Produkt zu weit; für die anderen sind die Werbebeschränkungen für ein Konsum-«Gut», welches abhängig macht und die Hälfte seiner Gebraucher umbringt, ungenügend. In einem Land, das den mehrheitsfähigen Kompromiss zum bevorzugten Werkzeug der Politik machte, hat der Entwurf aber gerade deswegen gute Chancen, angenommen zu werden. Die Mehrheit des Ständerates hat den Entwurf sogar an den Bundesrat zurückgewiesen mit dem Auftrag, die lückenhaften Werbeverbote zu streichen. Er betonte, gleichzeitig den Jugendschutz zu wollen, was unvereinbar ist. Erst das Endresultat wird wohl anzeigen, welchen Wert unsere Volksvertreter der Gesundheit von Mitbürgern und Weltbevölkerung beimessen.

Denn neben dem Jugendschutz geht es darum, einer Industrie Schranken aufzuerlegen, um die weltweite Tabakepidemie einzudämmen, die von ihr verursacht ist. Das von der WHO veranlasste Vertragswerk der Framework Convention Tobacco Control (FCTC) verpflichtet die Parteien, Regeln für Tabakprodukte einzuführen und deren Vertrieb, Promotion und Werbung auf nationaler und internationaler Ebene zu beschränken. Die FCTC hält fest, dass diese entwickelt worden

Tabelle 1: Die in der Schweiz heimischen Tabak-Multinationalen: Kennzahlen und Standorte (modifiziert, nach [5]). BAT = British American Tobacco, JTI = Japan Tobacco International, $\mathrm{PMI}=$ Philip Morris International.

\begin{tabular}{llll}
\hline & BAT & JTI & PMI \\
\hline Anzahl Mitarbeiter in der Schweiz & 1000 & 1300 & 3000 \\
\hline Zigaretten-Produktion in der Schweiz & $10 \mathrm{Mia}$. & $9,9 \mathrm{Mia}$. & $20 \mathrm{Mia}$. \\
\hline Umsatz weltweit, in CHF & $23 \mathrm{Mia}$. & $11,6 \mathrm{Mia}$. & $75,5 \mathrm{Mia}$. \\
\hline Marktanteil in der Schweiz & $40,5 \%$ & $16,3 \%$ & $42,3 \%$ \\
\hline Hauptquartier & Lausanne & Genf & Lausanne \\
\hline Produktionsstätten & Boncourt JU & Dagmersellen & Neuchâtel \\
\hline
\end{tabular}

sind, um Abhängigkeit zu erzeugen, und dass die Vertragsparteien sich verpflichten, die Gesundheit über den wirtschaftlichen Gewinn zu stellen. Ausserdem verpflichten sich die Länder, zu verhindern, dass Präventionsmassnahmen durch politische Manöver unterwandert werden. Die FCTC ist von beinahe allen Staaten ratifiziert worden. Obwohl die Schweiz sie unterzeichnet hat, ist sie auf dem europäischen Kontinent mit Andorra, Liechtenstein und Monaco die einzige Ausnahme.

\section{Liberale Wirtschaft im Kleinstaat}

Tabak war in der Schweiz nie staatliches Monopol. Vertrieb, Verkauf, Promotion und Werbung waren bis anhin im Lebensmittelgesetz geregelt. Das Wohlwollen des Staates gegenüber dem Tabak beginnt mit dem Zweiten Weltkrieg. Die Schweiz rationierte den Tabak damals nicht, er wurde im Gegenteil in die Massnahmen einbezogen, um der Bevölkerung genügend Nahrung zu gewährleisten. Um wegen erschwerter Importe die Produktion zu erhöhen, lobbyierte die «SOTA» (Kooperative für den Ankauf inländischen Tabaks). Deren Präsident Léon Burrus von der gleichnamigen Zigarettenfabrik in Boncourt argumentierte, dass der Tabak zur täglichen Nahrung gehöre, und zur Moral der Truppen beitrage. Obwohl Agrarland in Kriegszeiten dem Anbau von Weizen dienen sollte, wurde die Tabakanbaufläche vergrössert; die Produktion stieg von 1300 Tonnen im Jahre 1940 auf 2983 Tonnen im Jahre 1945, obwohl die Kultur einer Hektare Tabak sehr viel mehr Arbeitsstunden erfordert gegenüber lediglich 25 Stunden für eine Hektare Weizen [1]. Ausländische Tabakindustrien hatten sich für Niederlassungen in der Schweiz entschieden, 1913 Ed. Laurens und 1920 British American Tobacco (BAT). Philip Morris (PM) begann 1957 die "Marlboro» ausserhalb der USA in Neuchâtel zu produzieren. Marlboro war eine der ersten Zigaretten, die durch zugesetztes Ammoniak Nikotin rascher den Gehirnzellen zugänglich macht [2] und daher viel wirksamer abhängige Raucher erzeugt. Der Schweizer Markt war (und ist) attraktiv wegen seiner liberalen Gesetzgebung und weil er sehr verschieden- 
artige Konsumentensegmente mit hohem Einkommen zu testen erlaubt. Heute sind die grössten Zigarettenfabrikanten (ausser der nationalen chinesischen) bei uns heimisch: PM und Japan Tobacco International (JTI) mit ihren Welthauptquartieren und BAT (Tab. 1). Die Multinationalen arbeiten zusammen in der Organisation «Swiss Cigarette». Diese, wie auch die Schweizerische Volkspartei (SVP) und die Christdemokraten (CVP), unterstützen die Allianz der Wirtschaft für eine massvolle Prävention (AWMP), die von economiesuisse und dem Schweizerischen Gewerbeverband (SGV) gegründet wurde [3]. Hansueli Bigler, Direktor des SGV, ist mit der AWMP verbunden; als FDP-Nationalrat nimmt er die Interessen der Tabak-Multinationalen wahr, wenn er die «kleinen und mittleren Unternehmen" vertritt. Die Zusammenarbeit ist gut eingespielt; schon 1990 nannte PM den SGV «ihren Alliierten» [4].

Trotz der Verwurzelung stützt sich die Zigarettenindustrie kaum auf den heimischen Tabak; im Jahre 2014 wurden von knapp 200 Bauern (am Ende des Zweiten Weltkrieges waren es 6000) noch 997 Tonnen Tabak erzeugt. Dies entspricht wenigen Prozenten des Rohstoffes für die beinahe 40 Milliarden Zigaretten, welche 2014 zu über $80 \%$ in die Welt exportiert wurden [5]. Die Zahlen spiegeln die wirtschaftliche Realität, welche ohne staatliche Hilfe für den teuren schweizerischen Tabak anders aussehen würde. Vom Bund werden nämlich über die SOTA jährlich 14 Millionen CHF Subventionen in den Tabakanbau geleitet. Dies entspricht 0,3\% des Verkaufspreises der Zigaretten im Schweizer Markt, so viel, wie der Bund dem Tabakpräventionsfonds zugesteht [5]. Obwohl Absatzmarkt wie Tabakproduktionsland Schweiz unbedeutend sind, bietet das Zentrum Europas Vorteile. Unsere Gesetzgebung verbietet, hierzulande Produkte mit höherem Teer-, Nikotin- und Kohlenmonoxidgehalt zu verkaufen, als was für den europäischen Markt erlaubt ist. Diese Beschränkung gilt aber nicht für den Export [5]; so werden in die Ostländer, Asien und Afrika «Swiss Made»-Tabakprodukte exportiert, die durch höheren Nikotingehalt noch leichter als hier Abhängigkeit erzeugen und nirgendwo sonst in der westlichen Welt produziert werden dürften. Dies mit 18000 Tonnen importierten Tabaks und mit Hilfe der eidgenössischen Subvention. Schweizer Tabak ist so zu einem Segment der Volkswirtschaft geworden, das dem Export von Schweizer Käse oder Schokolade entspricht. Damit sind die Tabak-Multinationalen auch mit der schweizerischen Wirtschaft gut vernetzt. Neben dem SGV treten Gastrosuisse [6], economiesuisse [7], Werbung Schweiz [8] und das parlamentarische Lobbying für sie ein. Schweizer Advokaten vertreten sie in internationalen Streitigkeiten [9].
Diese Zusammenhänge erklären wohl, warum der Entwurf des TabPG einen Fortschritt der Tabakprävention nur vortäuscht, denn die Werbeverbote sind weit entfernt von denen Frankreichs. Sie würden die schweizerische Regelung der derzeitigen deutschen anpassen, von allen europäischen die unwirksamste (Tab. 2). Das nur für internationale Manifestationen geltende Sponsoringverbot ist gegenstandslos, da Tabakwerbung und -sponsoring wegen der Abkommen über internationale Sportsendungen verboten sind. Statt, wie von der FCTC vorgegeben, ein generelles, auch Zigarettenautomaten und Werbung an Verkaufsstellen umfassendes Verbot sieht der Entwurf Ausnahmen vor, welche das TabG zwecklos machen. Denn entgegen den Beteuerungen der Industrie, die Tabakwerbung diene der «Information der Konsumenten» und richte sich nicht an Jugendliche, ist ihre Funktion eine grundlegend andere [10]. Sie bezweckt, sowohl Gestik wie Tabakprodukte in der Gesellschaft gegenwärtig zu erhalten, um beim Nikotinabhängigen Rauchverhalten auszulösen und sie im kommerziellen und audiovisionellen Umfeld als banal erscheinen zu lassen. Dies ist besonders wirksam bei Kindern, welche wiederholte Signale als normal erfahren, und dies umso mehr, als sie ihnen regelmässig beispielsweise beim Einkaufen mit ihren Eltern vorgeführt werden.

\section{Freundlicher Föderalismus}

Das Prestige der Tabak-Multinationalen bei uns misst sich an ihren Palästen, in den besten Lagen von Lausanne und Genf, Nachbarn von Nestlé, dem Olympischen Komitee, Olympischen Museum, Internationalen Sportverband, der WHO und des UNO-Gebäudes. Vertrautheit zwischen Tabakindustrie und lokaler Politik lässt sich an den fast durchwegs positiven Antworten auf die Vernehmlassung zum TabPG erkennen. Die Argumente der Gemeinde Dagmersellen, Sitz der «Camel»-Fabrik von JTI, sind bezeichnend. Dagmersellen weist auf die "überdurchschnittliche Steuerleistung» hin. Der «Beitrag für die Schweizer Volkswirtschaft [...] inklusive Tabakanbau», wird hervorgehoben, und dass das Gesetz «explizit nur für Produkte gelten [sollte], welche für den Schweizer Markt bestimmt sind.» Dass der Bundesrat durch Verordnung gewisse Bestimmungen verschärfen dürfe, wird wegen der «Rechtssicherheit» abgelehnt. Viele Kantone stimmen $\mathrm{zu}$, «weil die Bestimmungen moderat sind». Für die Regierung des Kantons Zürich gehen die Regelungen des Sponsoring und der Werbung «zu weit». Die Werbung sollte auch «auf Gegenständen [erlaubt sein], die nicht mit Tabakprodukten in Zusammenhang stehen, wie z.B. Sonnenschirmen [...] und an den Verkaufsstellen». 
Tabelle 2: Regelungen im geltenden Gesetz (CH), im Entwurf zum TabPG (TabPG), verglichen mit den Bestimmungen Deutschlands (DE), Frankreichs (FR). Modifiziert nach [5] und Lungenliga Schweiz 2016. $X=$ verboten; $T$ = wie Tabak.

\begin{tabular}{|c|c|c|c|c|}
\hline & $\mathrm{CH}$ & TabPG & DE & FR \\
\hline Werbung speziell an Jugendliche & $\mathrm{X}$ & $\mathrm{X}$ & $\mathrm{x}$ & $x$ \\
\hline Werbung in Radio/TV & $\mathrm{x}$ & $x$ & $x$ & $x$ \\
\hline Gratisabgabe von Mustern & - & $x$ & $x$ & $\mathrm{x}$ \\
\hline Rabatte & - & - & $x$ & $x$ \\
\hline Plakatwerbung & - & $x$ & - & $x$ \\
\hline Werbung Kino & - & $\mathrm{x}$ & - & $x$ \\
\hline Promotion durch Hostessen & - & - & - & $\mathrm{x}$ \\
\hline Gedruckte Werbung und im Internet & - & $\mathrm{X}$ & $\mathrm{x}$ & $x$ \\
\hline Massenmailing an Erwachsene & - & - & - & $x$ \\
\hline Werbung auf Gebrauchsartikeln & - & $x$ & - & $x$ \\
\hline Sponsoring internationale Anlässe & - & $x$ & $x$ & $x$ \\
\hline Sponsoring nationale Anlässe & - & - & - & $x$ \\
\hline Werbung an Verkaufsstellen & - & - & - & $\mathrm{x}$ \\
\hline Abgabe an unter 18-Jährige & - & $x$ & $x$ & $x$ \\
\hline Zigarettenautomaten & - & - & - & $x$ \\
\hline Werbung für E-Zigaretten & - & $\mathrm{T}$ & $\mathrm{T}$ & $\mathrm{x}$ \\
\hline
\end{tabular}

Der Kanton Freiburg stellt sich als einzige Ausnahme präventionsfreundlich. Das TabPG sollte «die Tabakindustrie daran hindern, Kinder und Jugendliche [...] durch Manipulationstaktiken zum Rauchen anzustiften» und dies durch «ein globales Verbot von Werbung, Promotion und Sponsoring», da die Hälfte der Promotionsausgaben der Industrie in Werbung an den Verkaufsstellen investiert werde. Die Regeln sollten erlauben, sie der europäischen Direktive anzugleichen und durch Verordnung die neutrale Zigarettenpackung einzuführen.

Die Regierung des Kantons Neuenburg fordert eingangs, dass das Gesetz weitergehen sollte als der Entwurf: Der Entzug der Verkaufslizenz sollte denen drohen, welche sich nicht an das Mindestabgabealter halten. Dann aber geht er auf die «neuen Produkte der Substitution» ein, die im Entwurf «übermässig restriktiv angegangen werden, in einem Mass, welches drohe, sich im Bereich der öffentlichen Gesundheit kontraproduktiv auszuwirken». Der Entwurf nehme die Gelegenheit der «Entwicklung für das zukünftige Fortbestehen eines wichtigen Wirtschaftssektors» nicht wahr. Die «Lösung der Tabakepidemie auf Weltebene» wird mit «interessante[n] Perspektiven für den ganzen Wirtschaftszweig» verbunden. Die Werbeverbote sollten «noch gelockert werden für die neuen Produkte mit geringerem Risikoprofil», was den "doppelten Vorteil [hätte], die Werbebestrebungen der Industrie auf diese zu konzentrieren, und ihre Verankerung auf dem Markt beschleunigen würde».

Dies tönt wie ein Echo zu den Parolen von Professor Etter, Genf, und den Promotoren der elektronischen Zigarette. Der Zusammenhang wird transparenter, wenn man weiss, dass die Multinationalen massiv in diesen Markt investieren. Wirtschaftsförderung ist auch Anliegen des Kantons Waadt: «in Anbetracht der Wichtigkeit der Tabakindustrie für Arbeitsstellen, [sollten] die neuen, potentiell weniger schädlichen Produkte, wie die E-Zigarette mit oder ohne Nikotin, nicht denselben restriktiven Bedingungen unterstellt werden wie die konventionellen Zigaretten.» Werbeverbote sollten in der Kompetenz der Kantone bleiben.

Zusammenfassend unterstützen die Kantone den Entwurf, aber sie erheben zusätzlich für die Tabakindustrie Forderungen, die sie mit Föderalismus, Freiheit für Werbung und Förderung der Wirtschaft rechtfertigen. Die "potentiell weniger schädlichen Produkte» werden als für die weltweite Tabakprävention nützlich hingestellt. Dagegen wird nirgends auf Nikotin als Droge, noch auf die WHO-Rahmenkonvention hingewiesen.

\section{Wirtschaftsfreundliche Parteien}

Prävention wird von den politischen Parteien ignoriert. Die SVP «lehnt dieses bevormundende und einseitig ideologisch geprägte Gesetz ab. Der Trend der Zurückdrängung der Eigenverantwortung, der Subsidiarität, des Föderalismus, des Marktes, der Selbstregulierung und des Handelns nach Treu und Glauben kann nicht einfach weiter hingenommen werden». «Unter dem Vorwand des Jugendschutzes und der Prävention [...] resultiert der Ausbau des Staatsapparates durch weitere Kontroll- und Überwachungstätigkeiten [...]. Welche von den Gesundheitsaposteln als ungesund deklarierten Güter kommen als nächstes dran? Alkohol, Fett, Zucker, Salz oder Fleisch?» Pauschal wird Wirtschaftsfreiheit, Tabakindustrie, Genuss und individuelle Freiheit von Bürger und Konsument in einen Topf geworfen und in Schutz genommen: "Interessant ist hierbei der im Gesetz implizierte Generalverdacht, der eine gesamte Branche haltlos in Misskredit bringt [...] Tabak ist ein legales und auch kulturhistorisch anerkanntes Produkt, ein Genussmittel, welches in Eigenverantwortung konsumiert werden kann.»

Die Stellungnahme der FDP lehnt den Vorentwurf mit Werbefreiheit, Rechtsunsicherheit und Ungleichheit zwischen E-Zigarette / neueren Produkten und Tabakprodukten ab. Auch die Parteien in der Mitte des politischen Spektrums nennen diese Motive, wenngleich die BDP im Eingangsparagraph die «Verbesserung des Jugendschutzes», die Einführung des Mindestalters für den Erwerb von Tabakprodukten und die Regulierung neuer Produkte gutheissen. Die CVP findet, dass der Vorentwurf weit über das Hauptziel des Jugendschutzes hinausschiesst. 
Man stellt fest, dass die Parteien der Mitte und rechts der Mitte die FCTC, welche das Parlament ratifizieren sollte, keines Wortes würdigen. Eine unredliche Argumentation vermengt Werbeverbote für ein toxisches Produkt mit Verkaufsverbot und Verbrauch des Produktes. Dies entspricht der Kommunikationslinie des SGV, welcher in der Folge der Denigrationskampagne von 2008 gegen den Direktor des BAG, Prof. Zeltner, die Vertreter der Prävention als «Extremisten» apostrophiert und dem BAG die Absicht unterstellt, den Genuss allgemein, und mit ihm Alkohol, Fett, Salz und Tabak verbieten zu wollen [11].

\section{Schlussfolgerung}

Die Tabak-Multinationalen fühlen sich bei uns $\mathrm{zu}$ Hause. Sie profitieren von Subventionen, durchlässigen Gesetzen, und von einem Standort, der sie ihre toxischen Produkte exportieren lässt, wie dies sonst nirgendwo in der westlichen Welt möglich wäre. Sie gebrauchen den nationalen Verboten abgeneigten Föderalismus und die wirtschaftsfreundlichen politischen Parteien, welche internationale Konventionen ablehnen.

Auch der Bund und das BAG begünstigen die Tabakindustrie. Der Entwurf zum TabPG lässt vermuten, dass Einschüchterung schon während seiner Erarbeitung wirksam war. Denn von einem Gesundheitsminister hätte man erwartet, dass er einen den Forderungen der Gesundheit und den internationalen Empfehlungen entsprechenden Gesetzesentwurf vorstelle. Das hätte die Volksvertreter vor ihre Verantwortung gestellt und aufgezeigt, inwieweit sie durch Verwässerung des Entwurfes dem Druck der Zigarettenlobby nachgeben würden.

Das vorliegende TabPG lässt sich dadurch erklären, dass in der Schweiz wenig politischer Wille besteht, Transparenz zu schaffen, wo parlamentarische Entscheidungen gefällt werden. Die Parteifinanzierung, die Verbindungen zwischen Wirtschaft und Medien, sowie das Lobbying im Parlament sind keiner zwingenden Verpflichtung unterstellt, offen gelegt zu werden, und so ist die Rolle der Tabakindustrie als Einflussnehmerin tabu geworden. Wenn politische Weichen gestellt werden, beschränkt sich die Diskussion auf Argumente einer «liberalen» Wirtschaft. Die Experten der Prävention haben es dagegen schwer, ihre Argumente vernehmen zu lassen, denn die unter wirtschaftlichem Druck stehenden Medien vernachlässigen das Thema Prävention. Daher baut sich unter Bürgern und Publi- kum keine öffentliche Meinung auf. Diese wäre aber nötig, um den Parlamentariern bewusst zu machen, was mit dem chronischen Tabakkonsum für die Gesundheit der Menschen hier und weltweit auf dem Spiele steht.

Die PR-Massnahmen der Industrie und das Wohlwollen der Schweizer Zivilgesellschaft gegenüber den Tabakmultinationalen werden auf www.infosperber.ch als zweiter Teil dieses Artikels beschrieben.

\section{Literatur}

1 Martine Brocard: le Tabac en Suisse, une histoire qui dure. Swissquote, 3 Juillet 2015, 52-5.

2 RR Johnson: "Ammonia technology minute», B\&W 12 June 1989. Minn. Trial Exhibit 13,069. Zitiert in Gerard Dubois: le rideau de fumée. Kapitel «Une camisole chimique». Seuil, Paris. 2003.

3 Wirtschaft gegen übermässige Prävention. NZZ 30.05.2008.

4 Raymond Pantet, Director of public affairs. Philip Morris, July 12. 1990 (Bates Number 2024195742).

5 Thomas Angeli, Otto Hostettler : Das Geschäft mit dem Gift. Beobachter 22/2014, 23-9.

6 Die Resolution «für Lebensfreude und die Freiheit zu konsumieren", 4.6.1996 von GastroSuisse, entspricht weitgehend derjenigen des internationalen HO-Re-Ca-Kongresses, der von PM gesponsert wurde und an dem Florian Hew, bis 2010 Direktor von GastroSuisse, einer der Hauptredner war. Die Resolution wird in einem internen Dokument, datiert 19. Juni 1996, von PM so kommentiert: «Diese Resolution ist das Resultat der direkten Zusammenarbeit zwischen PM und GastroSuisse».

7 Thomas Pletscher, economiesuisse: Consultation on the exposure draft Tobacco Plain packaging Bill 2011 and Consultation Paper, to Department of Health and Ageing. Canberra Australia. May 192011.

8 PS Publicité Suisse und WS Werbung Schweiz sind seit 2016 unter dem Namen Kommunikation Schweiz / Communication Suisse zusammengeschlossen (www.ks-cs.ch). In seinem Kommunikationsrat ist PM durch Julian Pidoux vertreten. Unter den Mitgliedern der Auftraggeber finden sich PM, JTI und BAT. Der Präsident von KS/CS, Ständerat Filippo Lombardi, erklärt am 14.1.2016: «Das Hauptaugenmerk liegt zurzeit in der Politik [...] Der Ruf nach Einschränkungen der kommerziellen Kommunikation [wird immer lauter [...] [Es] braucht Mut zur Wahrhaftigkeit. Man muss die Unaufrichtigkeit, die sich hinter den Rufen nach Werbeverboten zeigt, ansprechen [...] Denn wenn man schon überzeugt ist, dass Produkte - weshalb auch immer - den Konsumentinnen schaden, soll man ehrlicherweise die Produkte verbieten - und nicht die Werbung dafür. Was legal zu kaufen ist, soll auch beworben werden dürfen.»

9 ZEIT ONLINE (9.8.2015): Wie Konzerne Staaten vor sich hertreiben. Philip Morris gegen Uruguay. Aufgrund eines Investitionsschutzabkommens von 1991 zwischen der Schweiz und Uruguay fordert die Multinationale vom Staat Uruguay einen Schadenersatz von 2 Mia. USD, was ca. 1/6 des Staatshaushaltes entspricht. Diese Forderung wird begründet mit den durch die Tabakverbote bedingten Handels- und Werbebeschränkungen. Gemäss Peter Niggli von Alliance Sud sieht das Abkommen Ausnahmen im Interesse der Allgemeinheit vor und ist «einseitig, da es den Investoren Rechte und den Gastländern Pflichten [einräumt]». Der Fall wurde im Juli 2016 vom Schiedsgericht der Weltbank zugunsten Uruguays entschieden.

10 Michela Canevascini, Hervé Kündig, Héloise Perrin, Claudia Veron: Observatoire des stratégies marketing pour les produits du tabac en Suisse romande, 2013-2014. Cipret Vaud 2014. (www.observa toire-marketing-tabac.ch

11 Noirjean H: Direkt zurück an den Absender. Schweizerische Gewerbezeitung 19.2.2016. 